\title{
Search for Signatures of Heavy Decaying Dark Matter with IceCube
}

\author{
The IceCube Collaboration ${ }^{\dagger}$ \\ $\dagger$ http://icecube.wisc.edu/collaboration/authors/icrc17_icecube \\ E-mail: stettner@physik.rwth-aachen.de, \\ hrvoje.dujmovicaicecube.wisc.edu
}

\begin{abstract}
Neutrino telescopes provide some of the best sensitivities for heavy decaying dark matter. With IceCube's observation of high-energy astrophysical neutrinos, interest in scenarios that could explain their origin as a result of the decay of long-lived heavy dark matter particles has risen. We present two dedicated experimental analyses to test this scenario of decaying dark matter with masses above $10 \mathrm{TeV}$. One analysis uses 6 years of IceCube data focusing on muon-neutrino tracks from the northern hemisphere and one analysis uses 2 years of cascade data from the full sky. The following contributions to the neutrino flux are considered: Atmospheric neutrinos, a diffuse astrophysical flux following a power-law spectrum and a potential flux of neutrinos produced in dark matter decays. The latter can be distinguished by its distinctive features in the energy spectrum (cut-off at half the mass of the DM-particle) and asymmetry of the arrival directions due to the DM halo of our galaxy. We present best-fit results and deduce lower lifetime limits on the order of $10^{28} \mathrm{~s}$ for dark matter masses above $10 \mathrm{TeV}$.
\end{abstract}

Corresponding authors: Jöran Stettner*1, Hrvoje Dujmovic ${ }^{2}$

${ }^{1}$ III. Physikalisches Institut, RWTH Aachen University, Germany

${ }^{2}$ Dept. of Physics, Sungkyunkwan University, Korea

35th International Cosmic Ray Conference - ICRC2017

10-20 July, 2017

Bexco, Busan, Korea

${ }^{*}$ Speaker. 


\section{PeV-Neutrinos and Dark Matter}

To this day, the origin of the high energy neutrino flux discovered by IceCube, with the purely atmospheric contribution being disfavoured at over $5 \sigma[1,2]$, remains unidentified [3]. We present two dedicated analyses to test the explanation of heavy $(m>10 \mathrm{TeV})$, unstable particles producing neutrinos in their decays as an alternative to bottom-up astrophysical acceleration scenarios. Since such heavy particles are predicted in many models which also aim to describe the particle nature of dark matter [4], the observation of high-energy neutrinos allows us to probe heavy decaying dark matter at the corresponding mass scales.

\section{Experimental Data}

The IceCube observatory is a cubic-kilometre neutrino detector installed in the ice at the geographic South Pole between depths of $1450 \mathrm{~m}$ and $2450 \mathrm{~m}$ [5]. Detector construction started in 2005 and finished in 2010. The neutrino reconstruction relies on the optical detection of Cherenkov radiation induced by secondary particles produced in neutrino interactions in the surrounding ice or the nearby bedrock.

For the presented analyses, two different event samples are used: The first analysis is based on six years of muon-neutrino data, i.e. track-like events from the northern hemisphere (no background from atmospheric muons) [2]. This event selection contains data from 2009 to 2014: The first two seasons consist of data taken with the partially installed detector, indicated by the labels IC59 and IC79. The season 2011 is labelled IC86-1 (full configuration) and the last three seasons are summarized as IC86-234 (full configuration and improved simulation). The second analysis uses two years of full-sky starting events. The data was taken during the period of June 2010 to March 2012 during the last year of construction (IC79) and the first year of full detector operation (IC86-1). The event selection is based on a previous study [6] which used a containment cut in order to achieve a high signal purity. In addition, a cut on the event track length is used to select only cascade-like events. These are events induced via an electron or tau neutrino or via a muon neutrino neutral current interaction. Due to the containment cut, the effective volume and thus the expected signal rate of the cascade sample is significantly smaller than that of the track sample. The cascade sample does, however, have a full sky coverage and an improved energy resolution which makes both analysis complementary to each other. The data samples do not share any events and the results can be treated as statistically independent.

\begin{tabular}{lcccc}
\hline & Numer of Events & Years & Sky coverage & Purity \\
\hline Track Sample & 352,294 & $6 y(2009-2014)$ & Nth. Hemisph. $\left(\Theta>85^{\circ}\right)$ & $99.7 \%$ \\
Cascade Sample & 278 & 2y $(2010-2012)$ & Full Sky & $90 \%$ \\
\hline
\end{tabular}

Table 1: Summary of the two event samples. 


\section{Analysis Methods}

To test whether the observed flux of high-energy neutrinos (partly) arises from heavy decaying dark matter, a forward folding likelihood fit is performed.

\subsection{Flux Components}

The flux components contributing to the total flux of neutrinos are of atmospheric and astrophysical origin. For a given neutrino energy and direction, the expected flux is calculated from the sum of the different flux templates.

Background components The largest source of background are conventional atmospheric neutrinos that originate from the decay of pions and kaons in cosmic ray air showers. These neutrinos are the largest background in both the track and the cascade sample. Atmospheric muons are not able to pass through the Earth, so they only contribute to the southern sky background. Another atmospheric background is expected from the prompt decay of charmed mesons, although the flux has not been detected yet [2]. A potential prompt contribution to the flux is therefore taken into account in both analyses as systematic uncertainty. Atmospheric neutrino flux predictions are taken from [7] and [8] for the conventional and prompt component (modified to account for the cosmicray knee), respectively.

Astrophysical Background: Cosmic-rays re-interacting in the vicinity of their production sites are expected to produce high-energy neutrinos [9]. A generic description that agrees well with the observed data is a diffuse flux following a single power-law energy spectrum [1, 2]. To allow for deviations from preceding results, the spectral index and the flux normalization are taken as free parameters in the presented analyses.

$$
\Phi^{\text {astro. }}=\Phi_{0} \times\left(E_{v} / 100 \mathrm{TeV}\right)^{-\gamma}
$$

Signal component Hypothetical heavy dark matter particles may decay into standard model particles (e.g. neutrinos [4]). The exact decay channel depends on the detailed nature of the dark matter particle. In the presented analyses, two similar hard benchmark channels are investigated (line-feature at $E_{v}=m / 2$ ): $D M \rightarrow Z^{0}+v_{\tau}$ (oscillated into $v_{\mu}$ ) for the analysis using track-like events and $D M \rightarrow H^{0}+v$ (flavour agnostic) for the analysis using the cascade sample, see figure 1. If there is a contribution from decaying dark matter to the high-energy neutrino flux, it can be identified with these benchmark channels. The derived lifetime limits can furthermore be translated to other decay channels.

The total expected flux from the hypothetical decay of dark matter consists of two components which are added up: dark matter particles decaying in the galactic halo and at cosmological distances [10]. Both share the same free model parameters in the fit, namely the lifetime and mass of the hypothetical dark matter particle.

The galactic component is expected to generate a flux of astrophysical neutrinos with two distinct features: An energy distribution which directly follows the decay spectrum $\frac{d N_{v}}{d E_{v}}$ and an angular distribution corresponding to the line-of-sight integral $J(\Psi)$. 


$$
\frac{d J^{\text {Halo }}}{d E_{V}}=\frac{1}{4 \pi m_{D M} \tau_{D M}} \frac{d N_{v}}{d E_{V}} \times J(\Psi) \quad J(\Psi)=\int_{0}^{\infty} \rho_{D M}(r) d s
$$

The Burkert profile is used as parametrization for the galactic halo with best fit parameters from [11], other halo profiles are studied as systematic uncertainty in Section 4.1. Compared to dark matter annihilation searches, the linear dependency on the dark matter density 3.2 makes the presented analyses less sensitive to the assumed halo profile.

The second dark matter subcomponent is coming from DM particles decaying at cosmological distances. The flux is assumed to be isotropic and the energy distribution follows the red-shifted decay spectrum. Using the $\Lambda \mathrm{CDM}$ model with parameters from [12], the expected flux is calculated from the original decay spectrum and the dark matter density in the universe $\Omega_{D M}$.

$$
\frac{d J^{\text {Cosm. }}}{d E_{v}}=\frac{\Omega_{D M} \rho_{\text {crit. }}}{4 \pi m_{D M} \tau_{D M}} \int_{0}^{\infty} \frac{1}{H(z)} \frac{d N_{v}}{d\left(E_{v}(1+z)\right)} d z \quad H(z)=H_{0} \sqrt{\Omega_{\Lambda}+\Omega_{m}(1+z)^{3}} .
$$

\subsection{Likelihood Fit and Test-Statistic Definition}

The likelihood fit aims to disentangle the different flux components by comparing $3 \mathrm{~d}$ flux templates with the experimental data as a function of energy, zenith angle and right-ascension. The templates are calculated from flux expectations which were obtained from a full detector simulation taking into account the same event selections as used for the experimental data.

The fit is performed by maximizing the Poisson likelihood to observe $n$ events if $\mu(\xi)$ are expected for a given set of fit-parameters $\xi$. The analysis of track-like events computes the likelihood per bin of $\log _{10}\left(E_{\mu}^{\text {reco. }}\right), \cos ($ zenith$)$ and right-ascension using Equation 3.4. The analysis of cascade-like events uses the unbinned likelihood (Eq. 3.5) obtained in the limit of an infinite number of bins. $N$ is the total number of observed events and $M=\int \mu d E d \Omega$ the total number of expected events.

$$
L(N ; \xi)=\prod_{i=1}^{b i n s} \mathrm{P}\left(n_{i} ; \mu_{i}\left(E_{j}, \phi_{j}, \theta_{j} ; \xi\right)\right) \quad(3.4) \quad L(N ; \xi)=\frac{\mathrm{P}(N ; M)}{M^{N}} \prod_{j=1}^{N} \mu\left(E_{j}, \phi_{j}, \theta_{j} ; \xi\right)
$$

Two fits are performed on the experimental data: The background hypothesis is the atmospheric and a diffuse astrophysical flux, the signal hypothesis additionally allows for a flux from decaying dark matter. Based on the two fits, a test statistic (Eq. 3.6) is calculated from the maximized likelihoods.

$$
T S:=2 \times \log \left(\frac{L\left(\hat{\phi}_{\text {astro }}, \hat{\gamma}, \hat{m}, \hat{\tau}\right)}{L\left(\hat{\phi}_{\text {astro }}, \hat{\gamma}, \tau=\infty\right)}\right) \geq 0
$$

\section{Experimental Results and Systematic Uncertainties}

The best-fit results are summarized in Table 2: Both show a decrease of the diffuse astrophysical flux if dark matter is considered in the fit (more so in the track analysis). However, the 


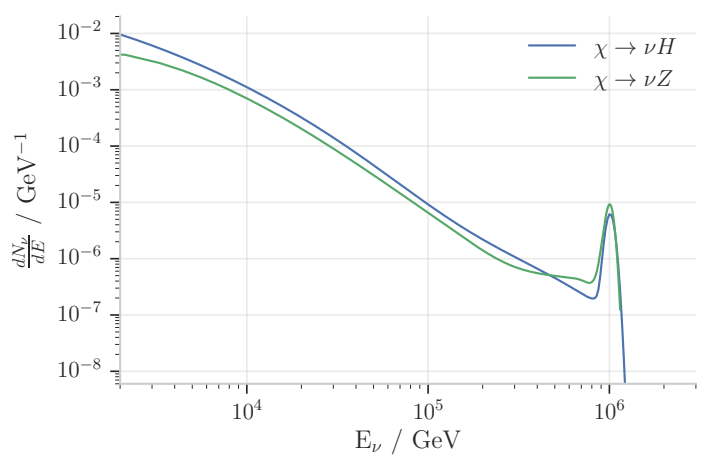

Figure 1: Benchmark neutrino spectra simulated from the kinematics and secondary processes ( $\mathrm{Spec}$ tra for the track-analysis from [13]). The simulated spectra are smeared out with a 5\% log-normal distribution for numerical stability and shown here exemplary assuming a $2 \mathrm{PeV}$ dark matter particle.

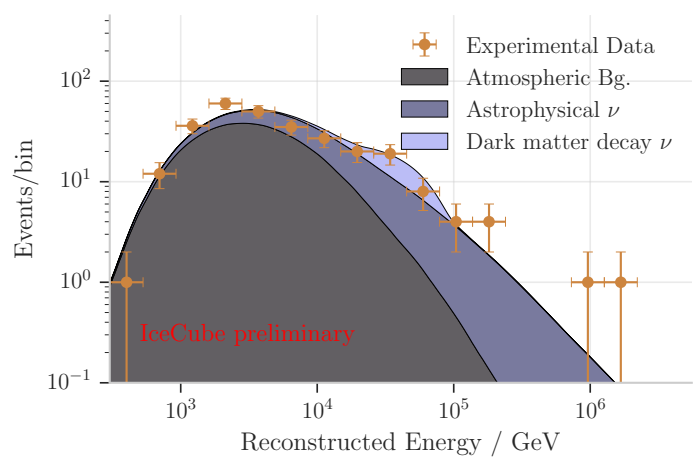

Figure 2: Cascade-Analysis: Best-fit energy distribution using the background plus dark matter decay signal hypothesis. The excess at $\sim 30 \mathrm{TeV}$ leads to the dark matter signal being fitted at a mass of 100 $\mathrm{TeV}$, it is well compatible with a background fluctuation though.

best-fitting dark matter mass and lifetime are different in both analyses and the track analysis observes a larger significance $(\mathrm{p}=3.5 \%)$. Figures 2 and 3 show the best-fit flux components together with the experimental data as a function of reconstructed energy.

While the significance of the result from the cascade-analysis is low $(p=55 \%)$ and a strong contribution from dark matter can thus be excluded, further investigations are needed for the trackanalysis. A real dark matter signal should exhibit a clear signature both in the energy distribution and the arrival directions, that is certain bins are expected to dominate the fit if a non-zero dark matter contribution is found. Figure 4 shows the significance per bin as a function of arrival directions revealing some differences between detector seasons; The events observed with the IC79 configuration indeed show a correlation between positive significance and the direction of the halo (similar but less strong observed in the seasons IC59 and IC86-1, not shown). The last three years of data (IC86-234), on the other hand, do not confirm this observation. A dark matter signal is expected to be constant in time and this anomaly may indicate a statistical fluctuation (Therefore, lower limits on the dark matter lifetime are computed in Sec. 5 for both analyses).

\begin{tabular}{lcc|cc}
\hline & \multicolumn{2}{c|}{ Track sample } & \multicolumn{2}{c}{ Cascade sample } \\
& Bg. & Signal+Bg. & Bg. & Signal+Bg. \\
\hline $\mathrm{m}_{\mathrm{DM}} / \mathrm{PeV}$ & - & 1.3 & - & 0.1 \\
$\tau_{\mathrm{DM}} / 10^{27} \mathrm{~s}$ & - & 22 & - & 8.2 \\
Astrophysical norm. $/ 10^{-18} \mathrm{GeV}^{-1} \mathrm{~cm}^{-2} \mathrm{sr}^{-1} \mathrm{~s}^{-1}$ & 0.97 & 0.16 & 2.17 & 1.63 \\
Astrophysical spectral index & 2.16 & 1.99 & 2.72 & 2.78 \\
$T S=2 \times \Delta L L H$ & $6.7 \rightarrow p=0.035$ & $3.4 \rightarrow p=0.55$ \\
\hline
\end{tabular}

Table 2: Best-fit parameters for both analyses. The quoted p-values are obtained from background pseudoexperiments. 

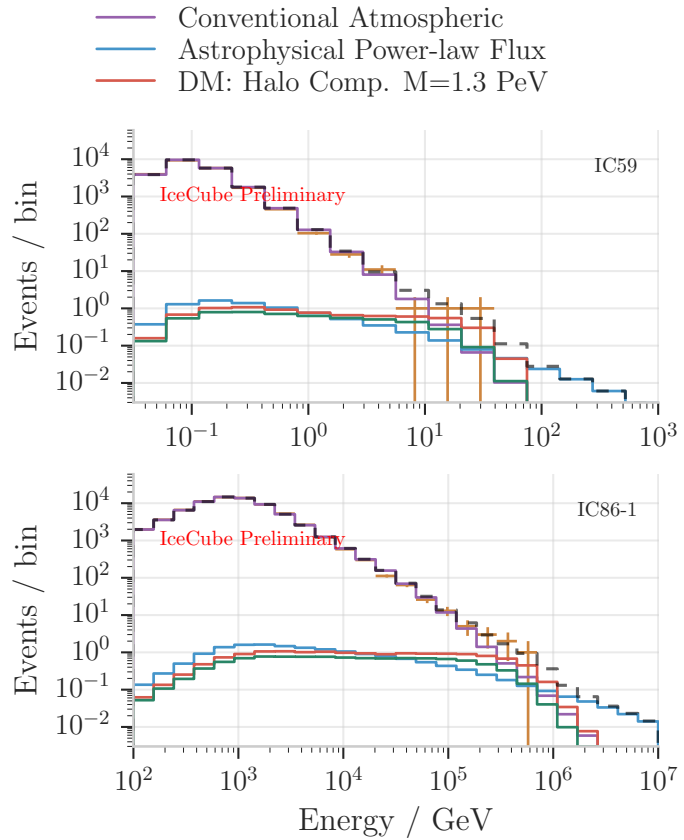
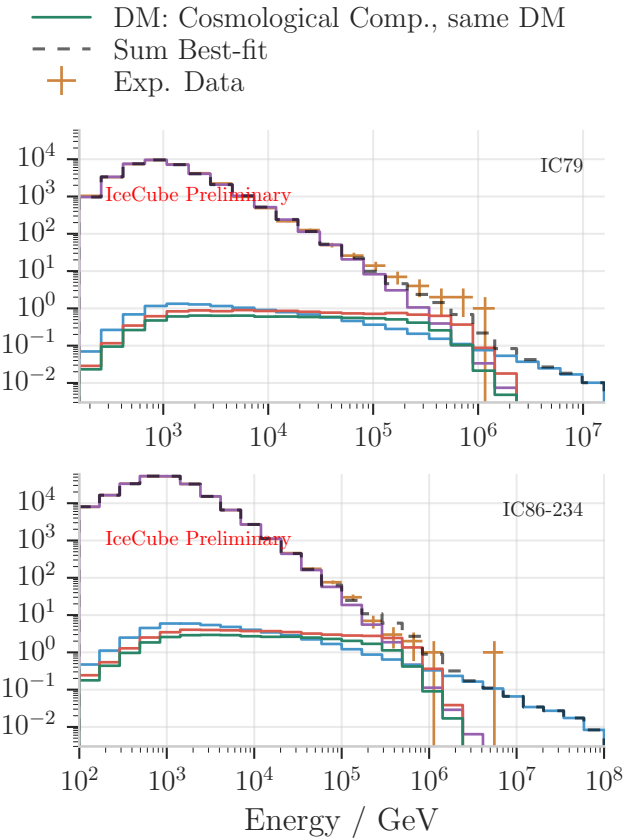

Figure 3: Track-Analysis: Best-fit energy distributions. While the low energetic events are well described by the conventional atmospheric component, the high energetic events are modelled by a combination of a weak diffuse flux and a component from decaying dark matter $\left(m_{D M}=1.3 \mathrm{PeV}, \tau_{D M}=2.2 \times 10^{28} \mathrm{~s}\right)$. The four histograms of the different detector configurations are shown separately for technical reasons but the fit was performed simultaneously with all seasons.

\subsection{Systematic Uncertainties}

To study the systematic uncertainties arising from imperfect modelling of flux components, ice properties and the behaviour of the detector, dedicated simulation datasets are used. In the trackanalysis, the impact of the following parameters is quantified and then parametrized as continuous nuisance parameters before the fit [2]: Normalization of the conventional and prompt atmospheric fluxes, cosmic-ray flux model uncertainties, relative contribution from Pion and Kaon decays to the atmospheric fluxes, several optical properties of the glacial ice and its modelling in the analysis and at last the optical efficiency of the detector modules. Their influence on the dark matter hypothesis turns out to be negligible because they are constrained by the low-energy region of the data with high statistics. In the cascade analysis, conventional and prompt atmospheric flux uncertainties [6], angular errors due to ice model uncertainties [14], 10\% uncertainty on the DOM efficiency and the impact of the finite simulation statistics are taken into account. The green dashed limit shown in figure 5 shows the impact of these systematics (no corresponding line for track-analysis because the impact is negligible). The overall effect is $10 \%-15 \%$ for DM masses below $5 \mathrm{PeV}$ and $<1 \%$ above.

A strong systematic uncertainty is the modelling of the dark matter flux prediction in both analyses. While the $\Lambda \mathrm{CDM}$-parameters used in the cosmological component were measured to reasonable precision $(\Delta \Omega / \Omega<3 \%)$, large uncertainties have to be taken into account for the halo component: The parameters of the Burkert profile are varied within their stated error ellipse (pre- 


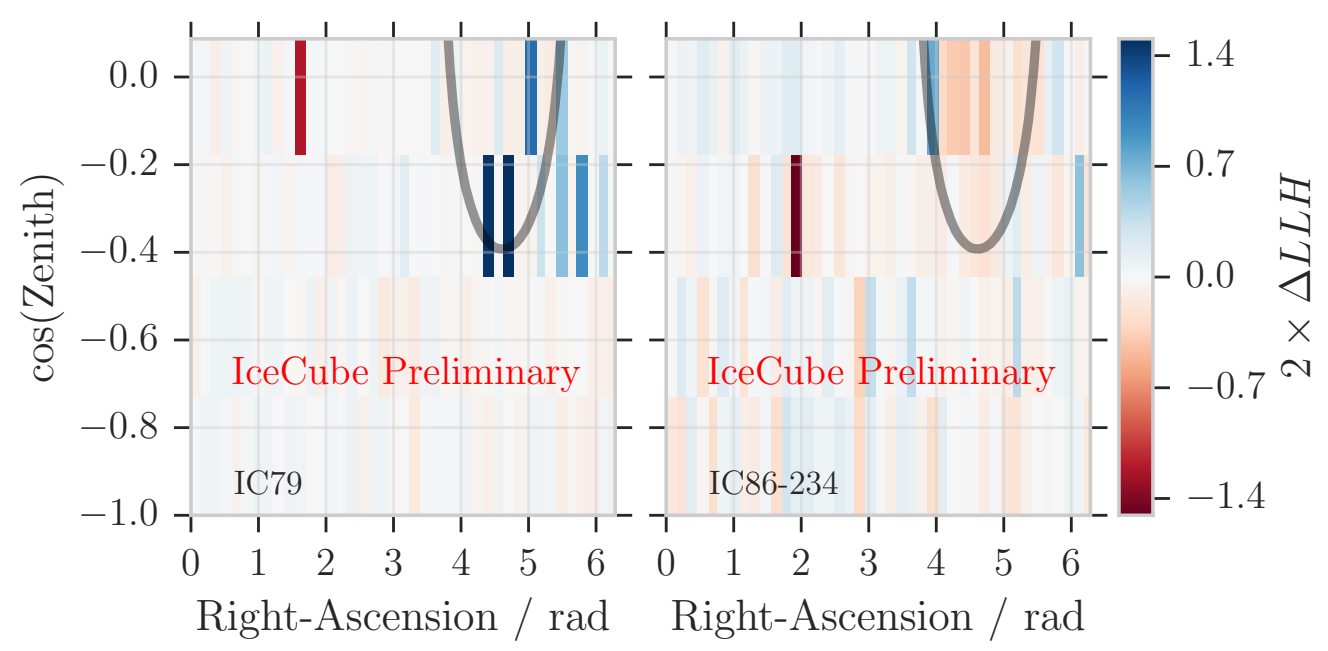

Figure 4: Track-Analysis: Significance per bin for the IC79 and IC86-234 configuration. Blue bins indicate where the signal hypothesis is preferred and vice versa. For comparison, the direction of the halo (half peak density) is shown as grey band.

ferred best-fit for $\beta_{2}=-0.5$, see discussion in [11]) and the maximal impact on the dark matter flux is evaluated (shaded bands around the limit curves in figure 5). This leads to a systematic uncertainty of approximately $10 \%$. For the track-analysis, it gives simultaneously an estimation of the uncertainty due to the choice of the Halo profile (NFW-profile prediction as second benchmark has smaller deviations.)

Last we note, that the tested hypothesis assumes a single power-law for the diffuse astrophysical flux. Deviations from the power-law assumption would have an effect on the results.

\section{Lower Limits on the DM Lifetime}

As a final result, limits on the lifetime of the dark matter particle are derived: Taking into account the observed differences between detector seasons, a (one-sided) lower limit relative to the background hypothesis is calculated as conservative approach in the track-analysis. The construction is based on Neyman confidence intervals [15], and relies on generated pseudo-experiments with dark matter signals injected. The resulting test-statistic distribution is compared to the experimental test-statistic value to calculate the limit contour at 90\%CL (red line in figure 5).

For the cascade analysis, the Feldman-Cousins approach [16] is taken to construct the acceptance intervals. Dark matter lifetimes, for which the observed test statistic is incompatible with the acceptance intervals, are excluded. As no significant signal has been observed, a lower limit on the lifetime is obtained $(90 \% \mathrm{CL}$, green line in figure 5). Figure 5 shows the limit contours from both analyses, also comparing to results from the IC22 and Fermi-LAT collaboration [17, 18].

In conclusion, two independent data samples containing 6 years of up-going tracks and 2 years of full-sky cascade events have been analysed. Although the fits in both analyses converge to a finite dark matter lifetime, they are consistent with background fluctuations. Excluded regions of the parameter-space are the currently best IceCube limit on the dark matter lifetime for dark matter masses above $10 \mathrm{TeV}$. 

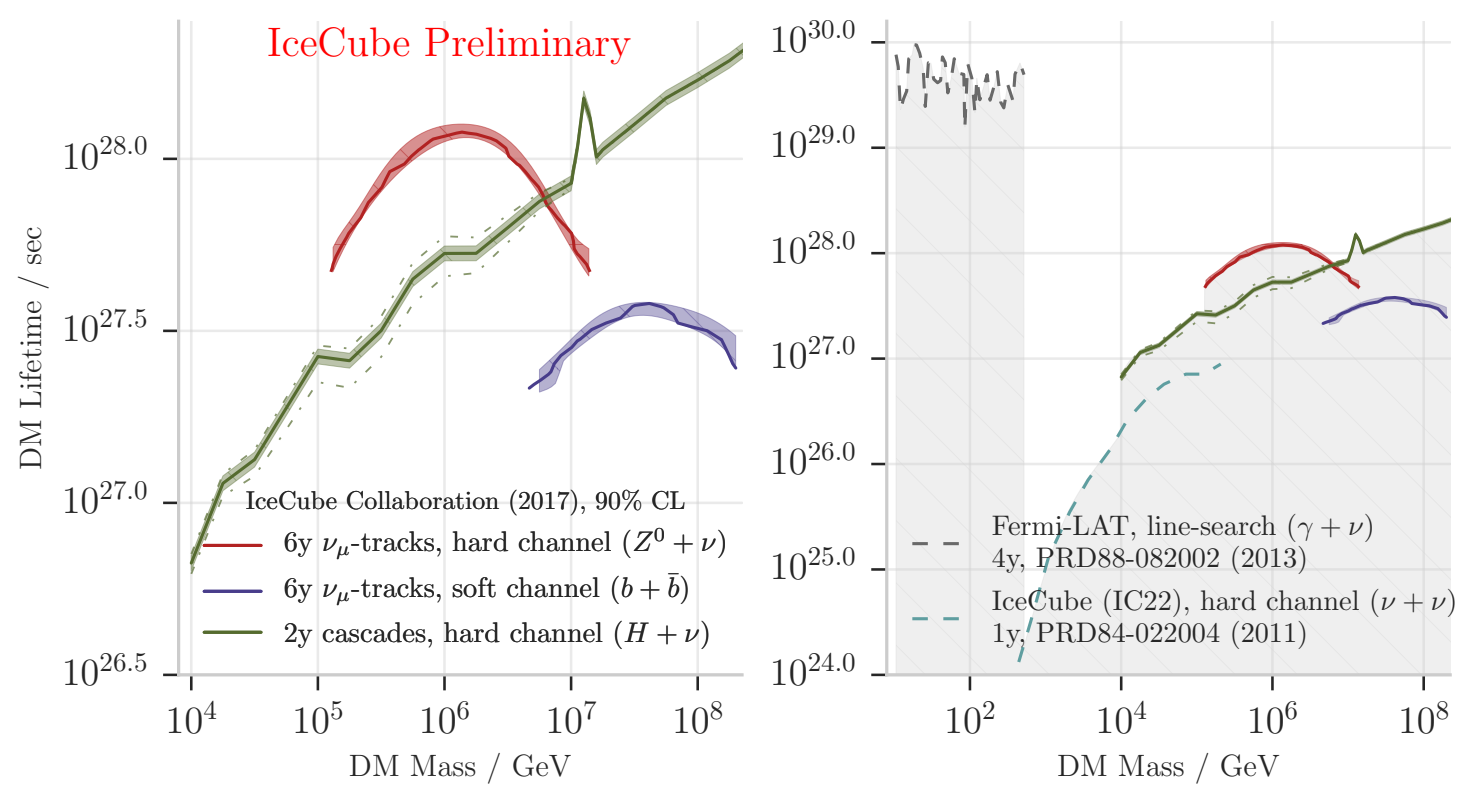

Figure 5: Lower limits on the dark matter lifetime as a function of the dark matter mass. Left: Results from this paper including systematics; For the track-analysis, additionally a converted limit assuming a soft decay channel $(b+\bar{b})$ is presented. The influence of detector systematics is negligible in the track-analysis (no dashed line). Right: Comparison to results from the Fermi and IceCube (IC22) collaborations.

\section{References}

[1] IceCube collaboration, M. G. Aartsen et al., Physical Review Letters 113 (2014) 101101.

[2] IceCube collaboration, M. G. Aartsen et al., The Astrophysical Journal 833 (2016) 3.

[3] IceCube collaboration, M. G. Aartsen et al., The Astrophysical Journal 835 (2017) 151.

[4] L. Covi et al., Journal of Cosmology and Astroparticle Physics (2010) .

[5] IceCube collaboration, Journal of Instrumentation 12 (2017) P03012.

[6] IceCube collaboration, M. G. Aartsen et al., Physical Review D 91 (2015) 022001.

[7] M. Honda et al., Physical Review D 75 (2007) 043006.

[8] Enberg, R. and others, Physical Review D 78 (2008) 043005.

[9] F. Halzen, Journal of Physics: Conference Series 337 (2012) 012050.

[10] A. Esmaili et al., Journal of Cosmology and Astroparticle Physics 1412 (2014) 054.

[11] F. Nesti and P. Salucci, Journal of Cosmology and Astroparticle Physics 1307 (2013) 016.

[12] Planck collaboration, P. A. R. Ade et al., Astronomy and Astrophysics 594 (2016) A13.

[13] J. Pepper, Ph.D. thesis, The University of Alabama, 2017.

[14] IceCube collaboration, M. G. Aartsen et al., arXiv:1705. 02383.

[15] J. Neyman, Philosophical Transactions of the Royal Society of London. Series A, Mathematical and Physical Sciences 236 (1937) 333-380.

[16] G. J. Feldman and R. D. Cousins, Physical Review D 57 (1998) 3873-3889.

[17] IceCube collaboration, R. Abbasi et al., Physical Review D 84 (2011) 022004.

[18] Fermi-LAT collaboration, M. Ackermann et al., Physical Review D 88 (2013) 082002. 